\title{
Stability of Electrical Coupling despite Massive Developmental Changes of Intrinsic Neuronal Physiology
}

\author{
Philip R. L. Parker, ${ }^{\star}$ Scott J. Cruikshank, ${ }^{\star}$ and Barry W. Connors \\ Department of Neuroscience, Division of Biology and Medicine, Brown University, Providence, Rhode Island 02912
}

Gap junctions mediate metabolic and electrical interactions between some cells of the CNS. For many types of neurons, gap junctionmediated electrical coupling is most prevalent during early development, then decreases sharply with maturation. However, neurons in the thalamic reticular nucleus (TRN), which exert powerful inhibitory control over thalamic relay cells, are electrically coupled in relatively mature animals. It is not known whether TRN cells or any neurons that are electrically coupled when mature are also coupled during early development. We used dual whole-cell recordings in mouse brain slices to study the postnatal development of electrical and chemical synapses that interconnect TRN neurons. Inhibitory chemical synapses were seen as early as postnatal day 4 but were infrequent at all ages, whereas TRN cells were extensively connected by electrical synapses from birth onward. Surprisingly, the functional strength of electrical coupling, assayed under steady-state conditions or during spiking, remained relatively constant as the brain matured despite dramatic concurrent changes of intrinsic membrane properties. Most notably, neuronal input resistances declined almost eightfold during the first two postnatal weeks, but there were offsetting increases in gap junctional conductances. This suggests that the size or number of gap junctions increase homeostatically to compensate for leakier nonjunctional membranes. Additionally, we found that the ability of electrical synapses to synchronize high frequency subthreshold signals improved as TRN cells matured. Our results demonstrate that certain central neurons may maintain or even increase their gap junctional communication as they mature.

\section{Introduction}

Gap junctions-plaques of ion channels that directly connect the cytoplasm of two cells-play many roles in brain function throughout life. During embryonic development, gap junction proteins are required for proper migration of cortical neurons along radial glia (Elias et al., 2007) and appear to be involved in metabolic coordination among neuronal progenitors (Bruzzone and Dermietzel, 2006). In early postnatal development many neurons in the brain, such as cortical pyramidal cells and thalamic relay cells, are clustered by gap junctional connections during the period when chemical transmission is being established (Connors et al., 1983; Lo Turco and Kriegstein, 1991; Kandler and Katz, 1995; Roerig and Feller, 2000; Dupont et al., 2006; Lee et al., 2006). In adulthood, the neuronal gap junctions in these regions apparently become rarer and limited to specific classes of neurons, such as cortical inhibitory interneurons and projection cells of the inferior olivary nucleus (for review, see Connors and Long, 2004). Adult gap junctions or electrical synapses allow for bidirectional current flow and mediate synchronous electrical activity between neurons. Interestingly, no studies have assessed

Received Sept. 23, 2008; revised May 7, 2009; accepted June 28, 2009.

This work was supported by Grants NS050434 and NSO25983 from the National Institutes of Health, the Brown Institute for Brain Sciences, and the Royce Fellowship Program. We thank Saundra Patrick for excellent technical assistance and Timothy Lewis for helpful comments on this manuscript.

*P.R.L.P. and S.J.C. contributed equally to this work.

Correspondence should be addressed to Scott J. Cruikshank, Department of Neuroscience, Division of Biology and Medicine, Box G-LN, Brown University, Providence, RI 02912. E-mail:Scott_Cruikshank@Brown.edu.

P. R. L. Parker's present address: Neuroscience Graduate Program, University of California, San Francisco, San Francisco, CA 94143 .

DOI:10.1523/JNEUROSCI.4568-08.2009

Copyright $\odot 2009$ Society for Neuroscience $\quad$ 0270-6474/09/299761-10\$15.00/0 the developmental time course of neuronal electrical coupling in areas that use this form of communication in adulthood.

Neurons of the thalamic reticular nucleus (TRN), which are GABAergic cells that receive neocortical input and have reciprocal connections with thalamic relay nuclei, are extensively connected by electrical synapses at juvenile ages [postnatal days (P) 12-21 in mice and rats] (Landisman et al., 2002; Long et al., 2004; Deleuze and Huguenard, 2006) and presumably into adulthood (Fuentealba et al., 2004). Small clusters of TRN cells are coupled by electrical synapses and show synchronous subthreshold and spike activity within these clusters (Long et al., 2004). TRN cells are also interconnected by inhibitory chemical synapses (Deschênes et al., 1985; Sanchez-Vives et al., 1997; Deleuze and Huguenard, 2006; Lam et al., 2006) although these synapses have remained elusive in paired whole-cell recordings (Landisman et al., 2002; Long et al., 2004).

TRN cells of rats and mice migrate into the thalamus by embryonic day 19 (Altman and Bayer, 1988; Mitrofanis and Baker, 1993; Ortino et al., 2003) and are in place within the nucleus by birth. During the first two postnatal weeks TRN cells are rapidly maturing morphologically, physiologically, and functionally. The cells enlarge, expand their dendritic arbors (Warren and Jones, 1997), and their axonal projections to the relay nuclei progressively mature (De Biasi et al., 1997). Intrinsic physiological properties of the cells also change drastically, especially spike-bursting characteristics and passive membrane properties (Warren and Jones, 1997). During this early postnatal period TRN cells communicate via GABAergic transmission that is depolarizing because of a high intracellular chloride concentration until around P9, after which GABA becomes clearly inhibitory (Pangratz- 
Fuehrer et al., 2007). No previous studies had tested whether TRN cells directly communicate via electrical synapses during early postnatal development.

To assess the prevalence of synapses between TRN cells during early postnatal development we performed dual whole-cell recordings from pairs of TRN cells aged P1 to P14. We found that TRN cells are extensively coupled by gap junctions from birth and the functional strength of these connections remains relatively constant across development because of offsetting decreases in input resistance and an upregulation of gap junctional conductance. Our data suggest that gap junction channels are homeostatically regulated during the development of some central neurons.

\section{Materials and Methods}

\section{Slice preparation and recording}

Brain slices containing the TRN were cut 320 $\mu \mathrm{m}$ thick in the somatosensory thalamocortical plane (Agmon and Connors, 1991) from P1 to P14 mice of the ICR strain. Slices were incubated at $32^{\circ} \mathrm{C}$ for $30 \mathrm{~min}$ after slicing, then held at room temperature until being moved to a $32^{\circ} \mathrm{C}$ submersion-type chamber for recordings. The bathing solution contained (in $\mathrm{mm}$ ) $126 \mathrm{NaCl}, 3 \mathrm{KCl}, 1.25 \mathrm{NaH}_{2} \mathrm{PO}_{4}, 2 \mathrm{MgSO}_{4}, 26$ $\mathrm{NaHCO}_{3}, 10$ dextrose, and $2 \mathrm{CaCl}_{2}$, saturated with $95 \% \mathrm{O}_{2} / 5 \% \mathrm{CO}_{2}$.

The TRN was identified by its characteristic shape and position, being bordered medially by the ventral basal thalamic nucleus (VB) and laterally by the internal capsule. Whole cell current clamp recordings were obtained from pairs of TRN neurons lateral to the VB (in the somatosensory sector of the TRN). Cells were visualized with infrared-differential interference contrast microscopy using a Nikon Eclipse E600FN and a CCD camera (MTI). Only pairs of cells whose somata were touching or within $\sim 2 \mu \mathrm{m}$ of each other were used for recording (Fig. 1D). Patch pipettes were made from $1.5 \mathrm{~mm}$ OD/0.86 mm ID glass (Sutter) and filled with (in $\mathrm{mm}$ ) 130 potassium gluconate, $4 \mathrm{KCl}, 2 \mathrm{NaCl}, 10 \mathrm{HEPES}$, 0.2 EGTA, 4 ATP-Mg, 0.3 GTP-Tris, 14 phosphocreatine-Tris ( $\mathrm{pH} 7.25$, 280-290 mOsm). Recordings were performed with Axoclamp 2B or MultiClamp 700B amplifiers (Molecular Devices). Series resistances were typically between 15 and $30 \mathrm{M} \Omega$ and were continually monitored and compensated throughout the recording sessions. Both series resistance and electrode capacitance were corrected conventionally using the built-in circuitry of the amplifiers. Voltages were corrected for a $14 \mathrm{mV}$ liquid junction potential off-line.

\section{Data analysis}

Recordings were acquired and analyzed using pClamp version 9 (Molecular Devices) and data were further analyzed in Excel (Microsoft) and Statview (SAS Institute). Statistical significance was defined as $p<0.05$ and determined using one-way ANOVA, Fisher's protected leastsignificant difference test, Mann-Whitney nonparametric tests, and linear regression analyses. All averages reported appear as mean $\pm \mathrm{SE}$.

Passive membrane properties. Resting membrane potential was measured within 5 min of breaking into whole-cell recording mode. Input resistance $\left(R_{\text {in }}\right)$ was calculated from small $(3-5 \mathrm{mV})$ voltage deflections induced by square hyperpolarizing current injections (averages of $20-40$ deflections). Estimates of the membrane time constant $\left(\tau_{\mathrm{m}}\right)$ were obtained by fitting an exponential function to these same hyperpolarizing voltage deflections. Input capacitance $\left(C_{\text {in }}\right)$ was calculated by dividing $\tau_{\mathrm{m}}$ by $R_{\text {in }}$. Spike properties were calculated using the first spike evoked by a small su-
Table 1. Summary of the number of TRN neuron pairs recorded for each age group ( $n=222$ cells total)

\begin{tabular}{llllllll}
\hline Age & P1-2 & P3-4 & P5-6 & P7-8 & P9-10 & P11-12 & P13-14 \\
\hline \# Pairs & 20 & 19 & 8 & 12 & 18 & 11 & 23 \\
\hline
\end{tabular}

prathreshold positive current step applied from a steady-state potential of approximately $-70 \mathrm{mV}$. The number of spikes per burst was obtained by holding the cell at a depolarized steady-state potential, then injecting a large hyperpolarizing current pulse ( $600 \mathrm{~ms}$ duration) to elicit a large hyperpolarization $(25-40 \mathrm{mV})$ and a subsequent rebound burst of action potentials.

Coupling coefficient. We calculated a steady-state coupling coefficient (CC) to estimate the functional strength of electrical coupling between cells. A $600 \mathrm{~ms}$ negative current pulse was injected into one cell of a coupled pair and resulting voltage deflections were measured in both cells. CC was defined as $V_{2} / V_{1}$ where $V_{1}$ refers to the voltage change in the current-injected cell and $V_{2}$ is the voltage change in the noninjected cell. The injected currents were adjusted so that $V_{1}$ was usually between 25 and $35 \mathrm{mV}$. The amount of injected current, which varied as a function of age (because of changes of $R_{\mathrm{in}}$ ), was between $-10 \mathrm{pA}$ and $-300 \mathrm{pA}$. The voltage changes were measured $300-500 \mathrm{~ms}$ after the start of the current step. The CC calculations were made for current injections into each cell in a pair and the two resulting values were then averaged. Cell pairs were considered to be electrically coupled if CC $>0.01$, which was the minimum that could be reliably detected under the conditions of our experiments.

Junctional conductance. To estimate the net conductance of gap junctional channels coupling two cells $\left(G_{\mathrm{J}}\right)$, we calculated junctional conductance as follows (Bennett, 1966):

$$
G_{\mathrm{J}}=1 / \frac{\left[\left(R_{\mathrm{in}} \text { cell } 1\right) *\left(R_{\mathrm{in}} \text { cell } 2\right)-(\text { transfer resistance })^{2}\right]}{\text { transfer resistance }},
$$

where transfer resistance was defined as the voltage response in cell 2 (when current is injected into cell 1) divided by the amplitude of the current step injected into cell 1 . This conductance calculation assumes a 
A
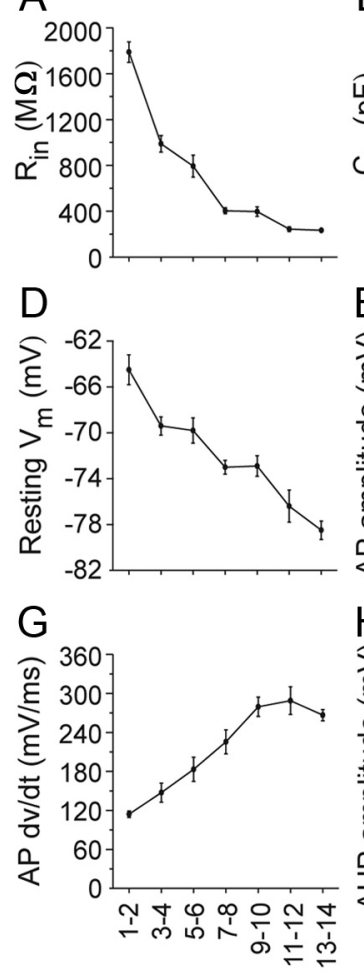

$\mathrm{B}$
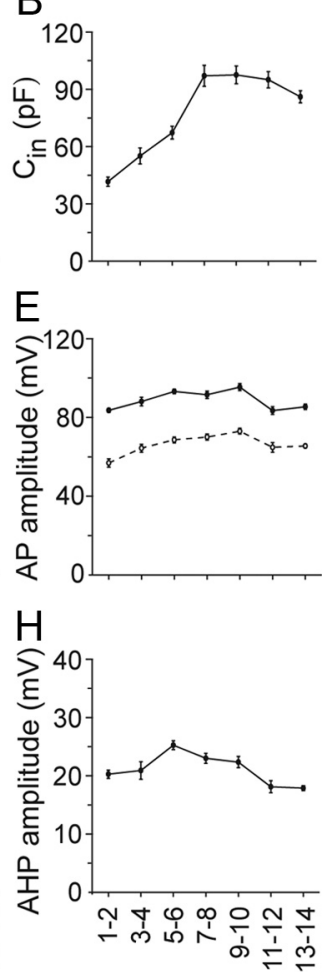

Postnatal Age (days)
C

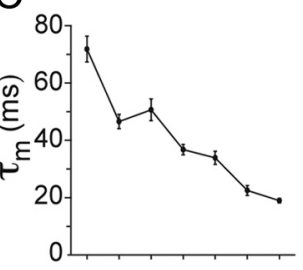

$\mathrm{F}$

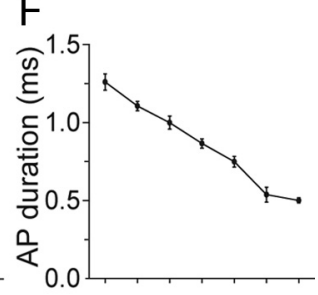

I

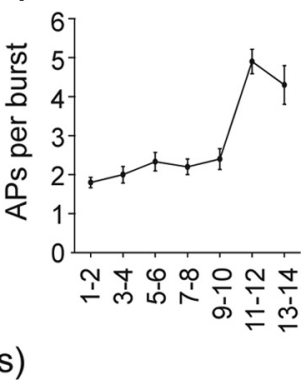

Figure 2. Changing intrinsic properties of TRN neurons across development. $A, R_{\text {in }}$ decreased nearly eightfold during the first two postnatal weeks. $B, C_{\text {in }}$ doubled. $C, \tau_{\mathrm{m}}$ affected by both $R_{\text {in }}$ and $C_{\text {in' }}$ decreased nearly fourfold. $\boldsymbol{D}$, The resting membrane potential hyperpolarized across ages, crossing the tonic/burst firing mode threshold. $\boldsymbol{E}$, Action potential (AP) amplitude was relatively constant across ages when measured from either the resting potential (solid circles) or from AP threshold (open circles). $\boldsymbol{F}$, AP durations, measured at half of the maximum amplitude, became briefer in older animals. $\boldsymbol{G}$, The maximum rate of rise of APs increased across age. $\boldsymbol{H}$, Amplitudes of the spike afterhyperpolarizations (AHPs) were relatively constant. $\boldsymbol{I}$, A sudden increase in the number of spikes per burst was seen between P9-10 and P11-12. Linear regression analysis revealed statistically significant effects of age $(p<0.05)$ for all of the intrinsic and spiking properties plotted here except AP amplitude when measured from baseline ( $\boldsymbol{E}$, solid line).

simple model consisting of two neurons with passive membranes coupled directly by a single junction and does not account for parallel conductance pathways via adjacent coupled neurons or dendritic cable properties (Amitai et al., 2002).

Frequency-transfer function. Frequency-transfer functions were derived from postsynaptic responses evoked by presynaptic, subthreshold sine-wave current injections through a range frequencies $(1,2,4,8,16$, 32,64 , and $128 \mathrm{~Hz}$ ). Baseline membrane potential was maintained at $-89 \mathrm{mV}$ and the induced presynaptic sine-wave voltages were $\sim 20 \mathrm{mV}$ (trough-to-peak) at all frequencies. CCs were calculated using $V 1$ and $V 2$, which in this case were the trough-to-peak amplitudes in the injected cell and the noninjected cell, respectively. Phase lag was measured by performing a cross-correlation on $V_{1}$ and $V_{2}$ and measuring the latency to the correlation peak.

Spikelets. Spikelets are electrical postsynaptic potentials resulting from presynaptic action potentials in cells connected by gap junctions. To measure spikelets, we evoked action potentials in one cell (nominally, the "presynaptic cell") with long current pulses ( $\geq 600 \mathrm{~ms}$ ) and recorded the resulting spikelets in the electrically coupled "postsynaptic cell." For each connected pair, between 25 and 175 spikelets were collected, aligned (using the presynaptic spikes) and averaged. Peak amplitudes of the averaged spikelets were measured relative to baseline voltages at the time of presynaptic spike onsets. Spikelet amplitudes were calculated this way for both cells in each pair and the two resulting values were then averaged to give a mean for the pair.

Spike cross-correlations. The ability of electrical synapses to synchronize action potentials was assessed by injecting coupled cells with enough

steady current to induce repetitive firing of $\sim 5-15 \mathrm{~Hz}$ in both cells. Conventional spike cross-correlograms were constructed from 15 to $30 \mathrm{~s}$ of spiking using pClamp and Excel. To facilitate comparisons among different cell pairs, the correlograms were subsequently normalized. First, spike counts for each bin were divided by total number of spikes in the reference cell (defined below), then baseline correlations were zeroed out by subtracting the mean value across all bins from each of the individual bins. Thus, the $y$-value for any given bin in the normalized cross-correlograms represents the probability of a spike occurring in that bin for one of the cells in a coupled pair (the target cell) given a single spike at time 0 in the second cell in the pair (the reference cell), with background activity subtracted out. The peak of the center bin of the correlogram, as well as the width of that peak at half its maximum value, were then measured.

\section{Modeling studies}

Simulations of gap junctional postsynaptic responses to presynaptic action potential waveforms were performed in simple models of P1 or P14 TRN cell pairs. For each pair, a single compartment postsynaptic cell was connected to a presynaptic voltage source by a gap junctional conductance, as described by the following equation:

$$
G_{j}\left(V_{\text {pre(t) }}-V\right)=G_{\mathrm{m}}\left(V-V_{\text {rest }}\right)+C_{\mathrm{m}} \frac{d V}{d t},
$$

where $G_{j}$ is the conductance of the gap junction connecting the presynaptic and postsynaptic cells, $V_{\text {pre(t) }}$ is the membrane potential of the presynaptic cell, $V$ is the membrane potential of the postsynaptic cell, $G_{\mathrm{m}}$ is the resting membrane conductance of the postsynaptic cell, $V_{\text {rest }}$ is the resting membrane potential of the postsynaptic cell, $C_{\mathrm{m}}$ is the membrane capacitance of the postsynaptic cell, and $t$ is the time in ms. Gap junctional conductances and postsynaptic membrane properties $\left(G_{\mathrm{m}}, C_{\mathrm{m}}\right)$, were set to the average values measured for P1 or $\mathrm{P} 14$ cell pairs recorded during the physiology experiments. Presynaptic action potential waveforms were varied and the effects on postsynaptic spikelet amplitudes examined. Voltages were updated in time steps of $0.05 \mathrm{~ms}$, which corresponded to the experimental sampling rate.

\section{Results}

\section{Intrinsic properties}

A total of 111 pairs of neurons were recorded from P1 to P14 (Table 1). Many of the intrinsic electrophysiological characteristics of TRN neurons changed drastically across ages, consistent with previous observations (Figs. 1, 2) (Warren and Jones, 1997). The most obvious intrinsic change was the nearly eightfold decrease in $R_{\text {in }}$ as animals matured $(1788 \pm 89 \mathrm{M} \Omega$ at P $1 ; 232 \pm 13$ $\mathrm{M} \Omega$ at P14) (Fig. 2A). The input resistance change was accompanied by a twofold increase in $C_{\text {in }}(42 \pm 2 \mathrm{pF}$ at P1, $86 \pm 3 \mathrm{pF}$ at P14) (Fig. 2B) and a nearly fourfold decrease in $\tau_{\mathrm{m}}$ (72 $\pm 4 \mathrm{~ms}$ at $\mathrm{P} 1,19 \pm 1 \mathrm{~ms}$ at P14) (Fig. 2C). All three membrane properties were strongly correlated with age $(r>0.60 ; p<0.0001)$. These changes suggest an increase in the size of TRN cells across 
development, which is supported by previous anatomical data (Warren and Jones, 1997).

The majority of cells across the entire P1-P14 age range were capable of two modes of firing-tonic and burst modes - when the steady-state membrane potential was moved above or below a threshold (approximately $-75 \mathrm{mV}$ ) (Fig. 1) (Jahnsen and Llinas, 1984; for review, see Huguenard and McCormick, 2007). However, age-dependent changes in the resting membrane potential affected the baseline mode of TRN firing. At P1 TRN cells had relatively depolarized resting potentials $(-64 \pm 1 \mathrm{mV})$ and as animals matured the average resting potential became more negative $(-78 \pm 1$ $\mathrm{mV}$ by P14) (Fig. 2D). Consequently, in young TRN cells, positive current steps applied from the resting potential resulted in tonic firing, whereas at older ages (after about P9) such positive current steps elicited bursts. Conversely, hyperpolarizing current steps from rest often elicited rebound bursts at young ages, whereas bursts could not be evoked by hyperpolarization from rest after about P9. Thus, as TRN cells mature, their basal firing mode appears to switch because of a hyperpolarization of the resting membrane potential. In addition, the number of spikes per burst increased across ages. From P1 until P6, most cells fired only one or two spikes per burst, whereas the later ages usually exhibited at least three spikes per burst $(1.8 \pm 0.1$ spikes/burst at $\mathrm{P} 1,4.3 \pm 0.5$ spikes/burst at P14; Figs. 1, 2I) (Warren and Jones, 1997). We observed examples of both typical and atypical bursts as described by Lee et al. (2007).

At least two other spiking properties of TRN cells changed significantly across ages: From P1 to P14, the maximum rateof-rise of action potentials more than doubled, whereas spike width decreased $>50 \%$ resulting in faster, narrower spikes (Figs. 1, 2F,G). In contrast, age related changes in spike and afterhyperpolarization amplitudes were relatively modest (Fig. 2E,H).

\section{Electrical synapses}

Electrical coupling (Fig. 3A) was very frequently seen at all ages. The incidence of coupling was high and relatively stable from P1 to P14 (50-100\% incidence) (Fig. 4A), suggesting extensive coupling between TRN cells at all ages. There was no apparent effect of age on CC $(r=$ $0.089, p=0.4371$ ) (Fig. 4B). In fact, cell pairs with robust coupling (CCs $>0.10$ ) were found in every age group. One ex-
A
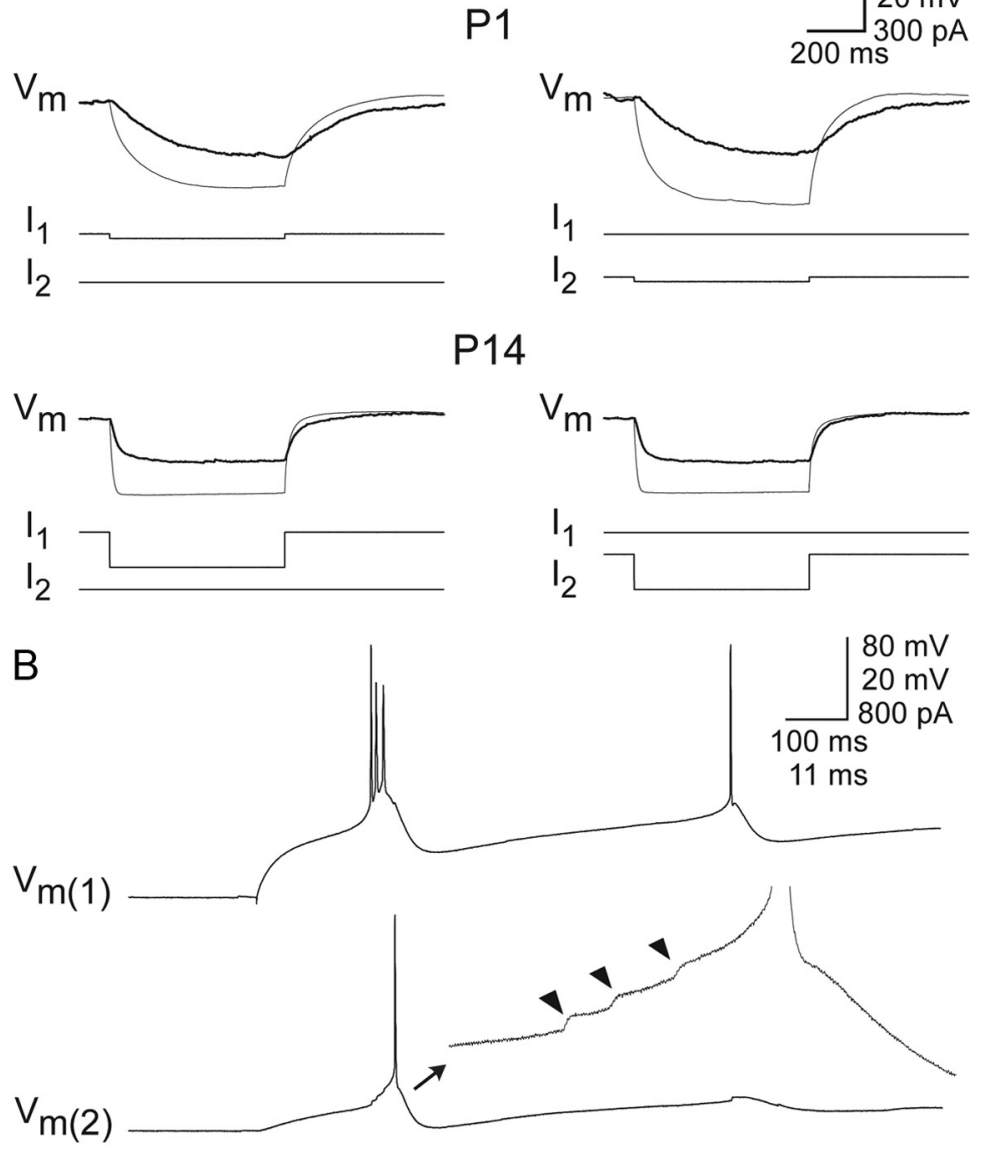

$I_{1}$

Figure 3. Electrical coupling in the immature TRN. $A$, Example of electrical coupling between pairs of cells in P1 (top) and P14 (bottom) mice. For each cell pair, traces corresponding to current injection into one cell (cell 1) are shown on left, and traces corresponding to injections into the other cell (cell 2) are on right. Voltage responses $\left(V_{m}\right)$ are superimposed for the injected cells (thin traces) and noninjected cells (thick traces). Current pulses $\left(I_{1}\right.$ and $\left.I_{2}\right)$ are displayed below their associated voltage traces. Negative currents caused strong hyperpolarizations of injected cells and smaller hyperpolarizations of noninjected cells (the latter as a result of current flowing through gap junctions). The time courses of voltage responses were faster in P14 than P1 cells. The voltage scale is $20 \mathrm{mV}$ for injected cells and $2 \mathrm{mV}$ for noninjected cells. $\boldsymbol{B}$, Strong electrical coupling in a P9 animal. Release of cell 1 from a hyperpolarized potential caused a rebound burst of action potentials in cell 1, depolarizing the noninjected cell (cell 2) via electrical coupling, ultimately causing a spike. Expanded trace: note summating spikelets (arrowheads) leading to an action potential in cell 2 . The $20 \mathrm{mV}$ and $11 \mathrm{~ms} \mathrm{scale} \mathrm{bar} \mathrm{values} \mathrm{apply} \mathrm{to} \mathrm{cell} \mathrm{2,} \mathrm{expanded} \mathrm{trace.} 0$ ther scale bar values apply to main traces.
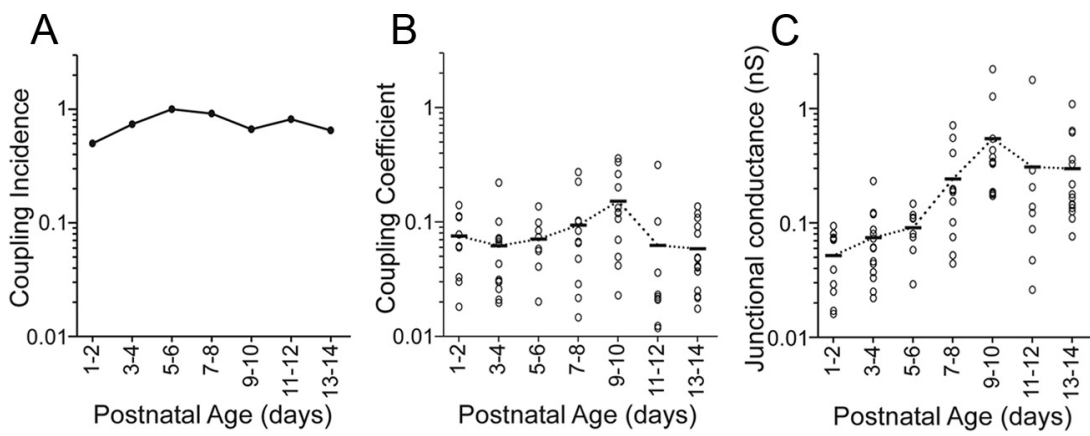

Figure 4. Properties of electrical coupling in the developing TRN. A, Incidence of electrically coupled pairs across ages was high and relatively stable. Values for each age group equal the (number of coupled pairs encountered) $\div$ (total number of pairs tested). $B$, Coupling coefficients changed very little across age. $\boldsymbol{C}$, There was a significant increase in junctional conductance with age $(r=$ $0.546, p=0.0001)$. $\ln B$ and $C$, circles are values for individual cell pairs and bars are age-group means. 

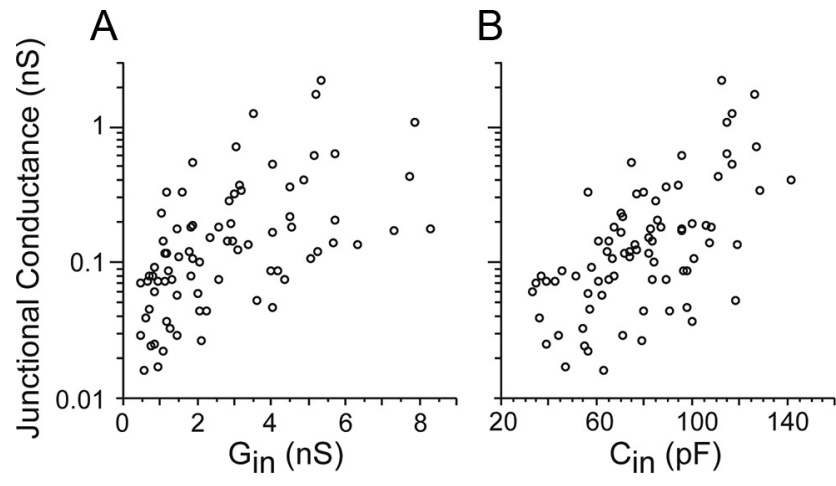

Figure 5. Relationships between passive membrane properties and gap junctional conductances. $\boldsymbol{A}$, Plot of input conductance $\left(G_{\text {in }}\right)$ versus gap junctional conductance. Circles are values for individual cell pairs (input conductances were averaged for the two cells within each pair). $\boldsymbol{B}$, Plot of input capacitance $\left(C_{\text {in }}\right)$ versus gap junctional conductance. There were strong correlations between gap junctional conductance and both of the membrane properties $(r \geq 0.43$, $p<0.0001$ for linear regression using raw values; $r \geq 0.567, p<0.0001$ using $\log _{10}$ transformed junctional conductances).

ceptionally strong example from a P9 animal is shown in Figure $3 B$, where a rebound burst in the injected cell triggered a spike in the noninjected cell.

Estimates of junctional conductance did show a significant effect of age ( $p<0.006$, ANOVA). However, in contrast to previous studies in other brain regions reporting decreases in gap junctions across early postnatal development (Dupont et al., 2006; for review, see Roerig and Feller, 2000), here the average junctional conductance was lowest at P1-2 and increased strongly with development (Fig. 4C). The mean conductances for the older age groups (P9-P14) were 6-10 times larger than that at $\mathrm{P} 1-2$. Functionally this large increase in gap junctional conductance was offset by a decrease in $R_{\text {in }}$ (Fig. $2 A$ ), thus yielding relatively constant CC. In fact, junctional conductance was well correlated with both age $(r=0.546, p=0.0001)$ and input conductance, the inverse of $R_{\text {in }}(r=0.567 p<0.0001)$ (Fig. $5 A$ ). There was also a strong correlation between junctional conductance and input capacitance, which is another cellular property that increases with age and acts to attenuate electrical postsynaptic potentials $(r=0.621, p<0.0001)$ (Fig. $5 B)$. Thus, as the TRN matures, its increasingly strong gap junctional conductances counteract the effects of increasingly leaky and capacitive nonjunctional membranes, stabilizing the strengths of electrical synaptic potentials across development.

\section{Subthreshold frequency transfer}

The ability of electrical synapses to transmit subthreshold signals of various frequencies (Gibson et al., 2005) was compared for young versus older TRN cell pairs $(n=4$ for P $1-4, n=5$ for P11-14). Injection of sinusoidal current pulses in one cell resulted in large sinusoidal voltage deflections in that cell and attenuated voltage responses in the coupled, noninjected cell (Fig. $6 A)$. The CC, which measures transmission of the voltage signal between the coupled cells, decreased as a function of increasing frequency. This frequency-dependent attenuation was more pronounced in the young (P1-P4) than in the older group (P11P14) (Fig. 6A, $B$, top). In addition, the phase lags of the signals were longer in the young cell pairs (Fig. $6 B$, bottom). Thus, whereas the steady-state coupling coefficients were relatively constant across ages, electrical transmission of time-varying signals actually improved in older TRN cells. These differences in signal transfer properties are likely caused by differences in the

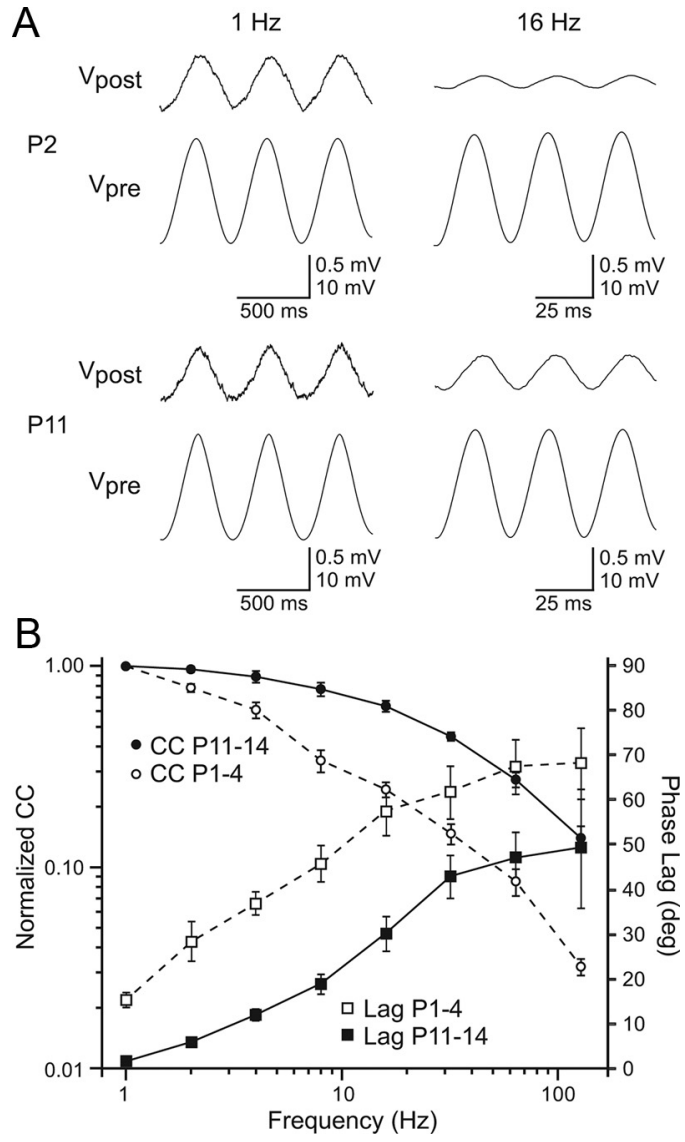

Figure 6. Age differences in subthreshold frequency transfer across electrical synapses. $\boldsymbol{A}$, Examples of subthreshold responses to sinusoidal current injections for P2 (top) and P11 (bottom) TRN cell pairs. Signals ( $1 \mathrm{~Hz}$, left) were transmitted across the electrical synapses more effectively than $16 \mathrm{~Hz}$ signals (right): compare ratio of postsynaptic to presynaptic voltage amplitudes $\left(V_{\text {post }}: V_{\text {pre }}\right)$. The high frequency attenuation in transmission is more pronounced in the P2 than the P11 cell pair. Peak-to-peak current injections: P2, $1 \mathrm{~Hz}=25 \mathrm{pA} ; \mathrm{P} 2,16 \mathrm{~Hz}=$ 90 pA; P11, $1 \mathrm{~Hz}=180$ pA; P11, $16 \mathrm{~Hz}=280$ pA. B, Plot of CC (left axis) and phase lag (right axis) at various frequencies of sinusoidal current injection for young $(\mathrm{P} 1-4, n=4)$ versus older $(\mathrm{P} 11-14, n=5)$ cell pairs. CC normalized to the $1 \mathrm{~Hz}$ value. Phase lag measured in degrees between the presynaptic and postsynaptic voltage responses across frequencies. The attenuation of C $C$ with increasing frequency was more pronounced in the younger group than the older group. Phase lags increased with frequency in both groups but were uniformly longer in the young group.

passive membrane properties between the two ages, as the average $\tau_{\mathrm{m}}$ of the younger cells was more than three times longer than that of the older group (young $\tau_{\mathrm{m}}=63 \pm 12 \mathrm{~ms}$, old $\tau_{\mathrm{m}}=19 \pm$ $2 \mathrm{~ms} ; p<0.0143$, Mann-Whitney).

\section{Spikelets}

The frequency transfer analysis might suggest that action potentials, which are predominantly high frequency signals, would be transmitted more effectively between older TRN cells than between younger ones. To examine this we evoked presynaptic action potentials and measured resulting postsynaptic spikelets in a sample of 35 coupled pairs from P1 to P14. Only cell pairs with medium coupling strengths were analyzed (CC between 0.04 and $0.14)$; pairs with weaker or stronger coupling were excluded to ensure accurate spikelet measurements and control for coupling strength across the age groups. Despite the improved frequency transfer functions in older pairs (Fig. 6), we found that spikelet amplitudes did not change significantly across ages $(r=0.08, p=$ 0.652 , Fig. $7 A, B)$. A decisive factor in this stability seemed to be 
shape of the presynaptic action potentials, which narrowed significantly with maturation (Figs. $2 F, G, 7 A$ ). In fact, when spikelet amplitudes were normalized by presynaptic spike width, a strong correlation with age emerged (linear regression, $r=0.50, p<0.003$ ) (Fig. 7C).

To further explore the effects of presynaptic action potential shape on spikelet amplitude in young and old TRN pairs, we varied action potential waveforms within computational models of electrically coupled cell pairs. The models were constructed using gap junctional and postsynaptic membrane properties derived directly from our experimental measurements (i.e., the average values for P1 and P14 from the experiments; Figs. 2, $4)$. Figure 7, $D$ and $E$, shows results from simulations using the models. When ageappropriate action potential waveforms were applied in the P1 and P14 models (i.e., wide spikes at P1 and narrow at P14) the resulting postsynaptic spikelets had approximately equal amplitudes for the two ages (Fig. 7D). In contrast, when a common presynaptic spike waveform (the average of $\mathrm{P} 1$ and $\mathrm{P} 14$ spikes) was applied in the models of younger and older coupled neurons, spikelets were much larger at $\mathrm{P} 14$ than at $\mathrm{P} 1$ (Fig. $7 E$ ); this was largely the result of the faster postsynaptic membrane time constant at P14.

Together the data indicate that spikelet amplitudes, like steady-state coupling coefficients, are relatively stable across age. The spikelet stability appears to be caused by a variety of offsetting changes that include narrowing of presynaptic action potentials and speeding of postsynaptic time constants as the TRN matures. Consistent with the overall kinetics, there was an obvious sharpening in the time course of the spikelets with maturation, including faster latencies to peak and shorter durations (Fig. $7 A, D, E$ ). The functional consequences of this sharpening remain to be explored.

\section{Spike cross-correlation}

To assess the ability of electrical synapses to synchronize spiking, constant current was injected into both cells of coupled pairs to induce similar steady firing rates of $\sim 5-15 \mathrm{~Hz}$, then the correlations of spike times were measured (Fig. 8A,B). Coupled TRN cells at all ages exhibited synchronous firing. Linear regression revealed a strong relationship between the amplitude of the correlogram peak and junctional conductance $(r=0.856 ; p<0.0001)$, and between the correlogram peak and CC $(r=0.715 ; p=0.0041)$ (Fig. $8 C)$. However, there was no significant relationship between the crosscorrelogram peak and age $(p>0.628)$, consistent with the coupling coefficient and spikelet results.
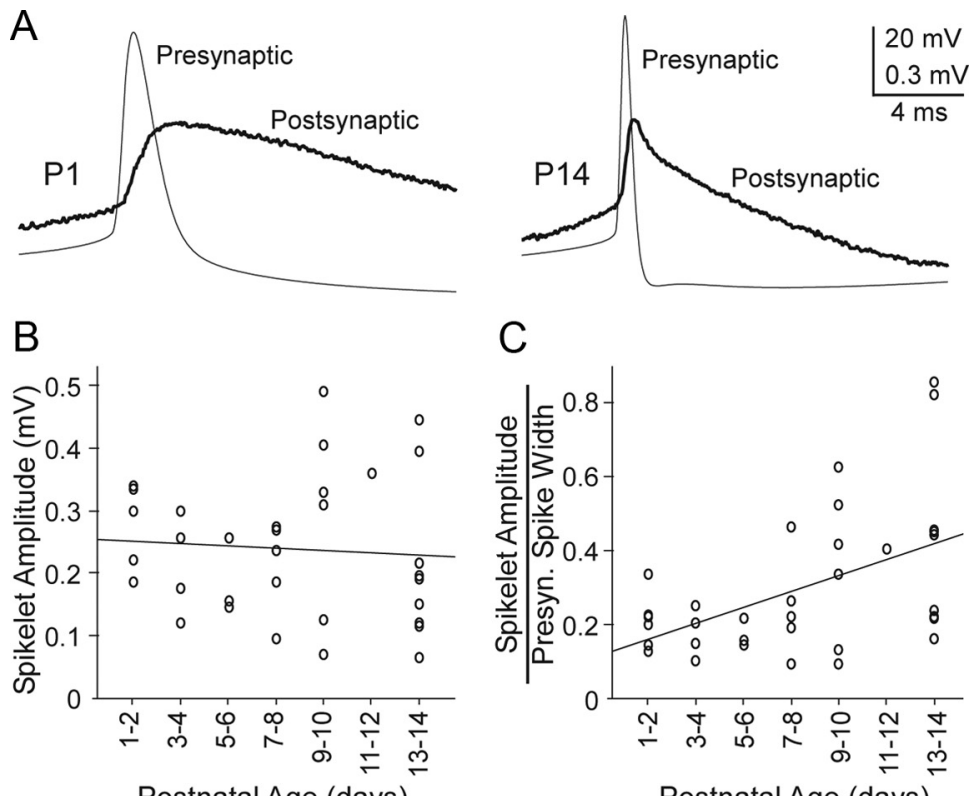

D Simulations: Default parameters for each age
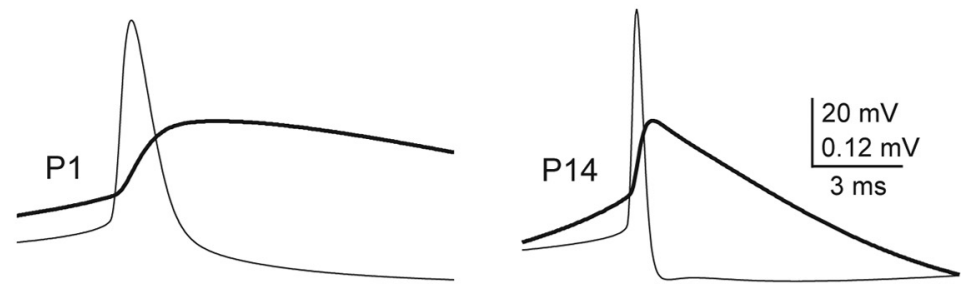

E Simulations: Presynaptic spikes held constant (otherwise default parameters)
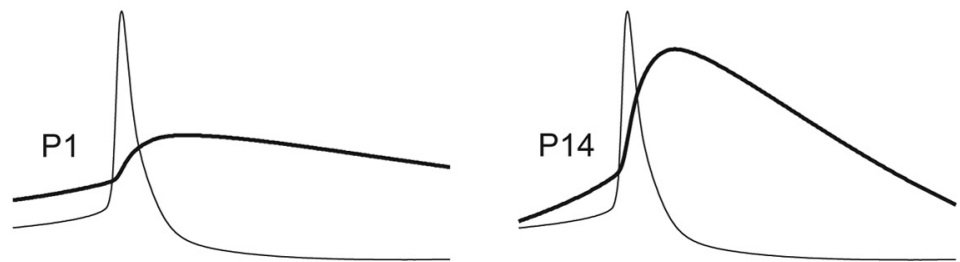

Figure 7. Spikelets amplitudes are stable across development and this depends on narrowing of action potentials. $\boldsymbol{A}$, Spikes (Presynaptic) and spikelets (postsynaptic) from representative P1 and P14 cell pairs (averages of $\sim 80$ sweeps each). Peak spikelet amplitudes are approximately equal. Steady-state coupling coefficients were 0.13 and 0.12 for the P1 and P14 pairs, respectively. Note the much wider presynaptic action potential at P1 than P14. B, Group data plotting raw spikelet amplitudes as a function of age, indicating no significant relationship ( $p=0.652, r=0.08$, linear regression, $n=35$ pairs). Each data point represents one pair of cells. $C$, When spikelet amplitudes were normalized to presynaptic spike width (at half-amplitude, in ms), a strong correlation with age emerged $(r=0.50, p<0.003)$. D, Results from model cell pairs connected by gap junctions. The presynaptic spike waveforms were the same as those in $\boldsymbol{A}$ (which are representative for their respective ages). Gap junctional conductances and postsynaptic $G_{\text {in }}$ and $C_{\text {in }}$ were set to average values recorded at $\mathrm{P} 1$ and $\mathrm{P} 14$ (from Figs. 2 and 4). Spikelet amplitudes were similar in the P1 and P14 model cells, and the spikelet shapes at each age were similar to those of the real spikelets shown in $A$. $E$, To test the effect of action potential shape, a common presynaptic spike waveform (the average of the P1 and P14 spikes from D) was applied in both the $\mathrm{P} 1$ and $\mathrm{P} 14$ models. Under these conditions, spikelets were much larger at P14 than at P1, indicating that narrowing of spike width normally contributes to stability of spikelet amplitudes as cells mature.

\section{Inhibitory synapses}

We encountered only 5 inhibitory synaptic connections among 90 pairs of neurons tested, and these occurred in 4 of the cell pairs - one pair had reciprocal inhibitory connections (Fig. 9). This yields a $2.8 \%$ incidence of inhibitory connections, i.e., 5 among 180 potential connections. The connections were seen at P4, P5, P7, and P8 (reciprocal pair). Presumably these were at least partly $\mathrm{GABA}_{\mathrm{A}}$ receptor-mediated responses because they were hyperpolarizing from the resting potentials and reversed around $-89 \mathrm{mV}$, near the expected equilibrium potential for $\mathrm{Cl}^{-}$ 
A
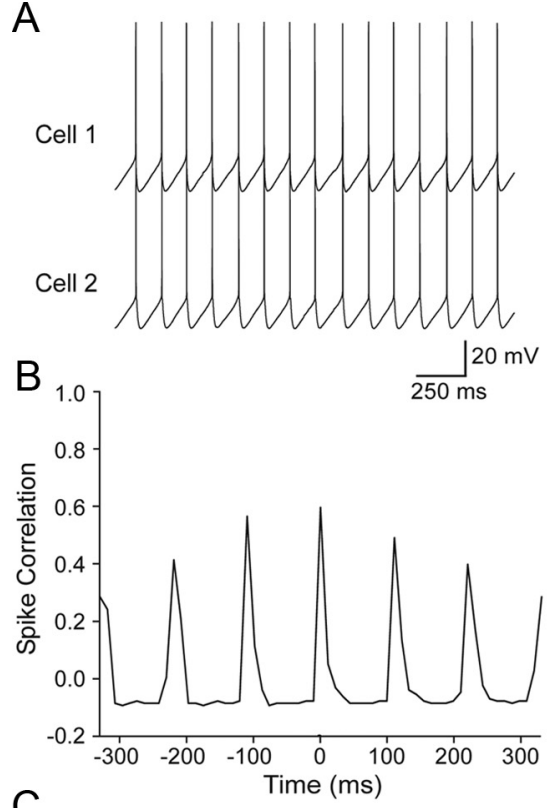

C

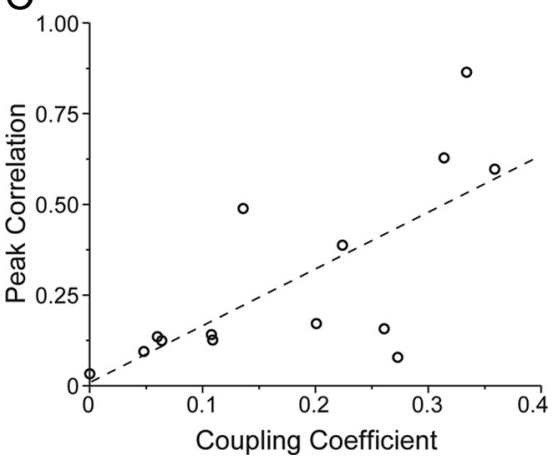

Figure 8. Spike synchrony between pairs of coupled TRN cells. $A$, Example $P 9$ cell pair exhibiting spike synchrony during simultaneous steady-state current injection (80 pA). B, Action potential cross-correlogram from the cell pair in $\boldsymbol{A}$ (bin width, $11 \mathrm{~ms}$ ). Fifteen seconds of spiking data were acquired for the plot shown here. Note the large central peak indicating strong synchrony. C, The peak of the AP correlation (measured at the peak closest to zero lag) was plotted as a function of the steady-state CC for 14 cell pairs at a variety of ages (from P1 to P14). There was strong positive relationship between $C($ and AP correlation ( $p=0.0064$; regression line shown). Junctional conductance was likewise strongly related to AP correlation ( $p=$ 0.0011; data not shown).

under these recording conditions, and because of their fast initial time courses. Some contribution from $\mathrm{GABA}_{\mathrm{B}}$ receptormediated responses cannot be ruled out. The average IPSP amplitude was $-1.1 \mathrm{mV}$ from rest. All of the inhibitory connections occurred between electrically coupled cells, however there was no obvious relationship between strength of coupling and the existence or strength of inhibition. In some strongly connected pairs, postsynaptic responses to presynaptic spike trains resulted in fast electrically mediated PSPs (spikelets) summating with slower hyperpolarizing IPSPs, resulting in a progressive hyperpolarization of the peak of each spikelet, creating a saw-tooth-like waveform (Fig. 9B). In general the inhibitory synapses depressed easily, and at least 15-20 s of recovery was required between stimuli to maintain stable responses.

\section{Discussion}

Our results demonstrate that neurons of the TRN maintain functionally stable electrical coupling from the day of birth through the first two postnatal weeks. Over the same period, the intrinsic membrane properties of TRN neurons change dramatically. The data imply that the conductances of neuronal gap junctions must increase sharply to compensate for increasingly leaky nonjunctional membrane.

\section{Development of intrinsic properties}

The intrinsic membrane properties of most neurons change considerably as they develop (Spitzer and Ribera, 1998; Moody and Bosma, 2005). We found that the intrinsic electrophysiological properties of TRN cells changed considerably during the first two postnatal weeks, in agreement with an earlier study (Warren and Jones, 1997). Most noticeable were changes in $R_{\mathrm{in}}, C_{\mathrm{in}}$, and $\tau_{\mathrm{m}}$. The drop in $R_{\text {in }}$ is probably attributable in part to increasing cell size during development (Warren and Jones, 1997). In addition, it is likely that leak potassium channels increase; this is implied by a negative shift in resting membrane potential, and because whole-cell capacitance - a measure of total membrane areaincreased only about twofold, whereas input conductance increased approximately eightfold. Gap junctions likely also contribute to the developmental decrease in input resistance. The average junctional conductance increased sixfold from P1 to P14, and this could result in substantial increases in total input conductance.

Spiking properties also changed. Action potentials in maturing neurons acquired faster rates of rise and shorter durations. Furthermore, the number of spikes within each intrinsic burst response increased sharply after about $\mathrm{P} 10$. Faster $\tau_{\mathrm{m}} \mathrm{s}$ as well as changes in voltage-dependent cation conductances may contribute to the spike effects in developing cells. Interestingly, only modest decreases were seen in the potassium-mediated spike afterhyperpolarization, and the low threshold calcium spike which provides the underlying voltage envelope of thalamic bursts (Jahnsen and Llinas, 1984; Huguenard and Prince, 1992; Lee et al., 2007) was observed in even the youngest animals.

\section{Development of inhibitory synapses}

There is considerable structural and physiological evidence that local GABAergic connections exist in the TRN (Deschênes et al., 1985; Ohara, 1988; Sanchez-Vives et al., 1997; Deleuze and Huguenard, 2006; Lam et al., 2006). Nevertheless, published data from paired recordings had not previously revealed any monosynaptic inhibitory connections (Landisman et al., 2002; Long et al., 2004). Here we observed a $2.8 \%$ incidence of GABAergic connections. This may be an underestimate resulting from confounding factors such as processes being cut during slice preparation (Deleuze and Huguenard, 2006).

The IPSPs observed in our TRN cells were hyperpolarizing, presumably because of the low $\left[\mathrm{Cl}^{-}\right]$in our micropipette solution. A recent report found that GABAergic responses in nondialyzed TRN cells are depolarizing from resting potential at ages younger than P8 (Pangratz-Fuehrer et al., 2007). Thus, it would be expected that in young animals in vivo, depolarizing PSPs from GABAergic connections could summate with electrically transmitted spikelets, leading to large, long lasting depolarizations. It has been suggested that such depolarizations provide trophic influences (De Biasi et al., 1997; Pangratz-Fuehrer et al., 2007).

\section{Development of electrical synapses}

Electrical synapses are a major feature of the juvenile TRN circuitry (Landisman et al., 2002; Long et al., 2004), and they are probably also widespread in adult TRN (Fuentealba et al., 2004; Deleuze and Huguenard, 2006; Blethyn et al., 2008). Our results show that electrical coupling is also ubiquitous among TRN cells 
during the first two postnatal weeks. The incidence and functional strength of coupling (i.e., CC and spikelet amplitudes) during this period were relatively constant and similar to previous reports for juvenile mice (Long et al., 2004). However, our data also indicate that this constancy of coupling is achieved by an upregulation of junctional conductances which offset maturational changes in passive membrane properties such as input resistance and capacitance. Our results do not reveal whether the developmental changes in membrane properties have any direct role in regulating gap junction properties. However, a linkage is suggested by the fact that junctional conductance correlates slightly better with input conductance and capacitance than it does with age.

Enhancement of gap junctional conductance could be achieved in many ways. Neurons might increase single channel conductances or the open probability of connexin channels, perhaps by channel phosphorylation or some other posttranslational modification (Cachope et al., 2007). Numerous studies have suggested that coupling between central neurons can be modulated by neurotransmitterinduced actions (for review, see Roerig and Feller, 2000; Connors and Long, 2004). Studies in the TRN have been few, but work in our laboratory showed that coupling between juvenile TRN cells is persistently decreased by the transient activation of metabotropic glutamate receptors (Landisman and Connors, 2005). In the developing neocortex several neurotransmitters, including glutamate, norepinephrine, dopamine, serotonin, and acetylcholine have been implicated in the regulation of neuronal dye coupling (Rörig and Sutor, 1996; Montoro and Yuste, 2004) or electrical coupling (Zsiros and Maccaferri, 2008) by initiating signaling cascades leading to phosphorylation. Decreases in dye coupling and Cx36 expression are seen during early development of the hypothalamus, and these are action potential-dependent (Arumugam et al., 2005). Alternatively, neurons could regulate the turnover of connexins and gap junction channels, and perhaps increase the number of channels per gap junction or the total number of gap junction plaques connecting pairs of neurons. It seems likely that a combination of these mechanisms underlies the enhancement of coupling conductance during TRN development (Condorelli et al., 1998; Arumugam et al., 2005; Moreno and Lau, 2007).

\section{Functions of electrical synapses}

Neuronal gap junctions are pervasive in structures of the CNS during development. As our results demonstrate, the TRN is no exception. In fact, during the first postnatal week the other major category of thalamic neurons - thalamocortical relay cells-are also coupled by gap junctions (Lee et al., 2006), as are their targets, pyramidal cells and spiny stellate cells of the neocortex (Connors et al., 1983; Lo Turco and Kriegstein, 1991; Yuste et al., 1992). Unlike the principal neurons of both rodent thalamus and neocortex, however, TRN neurons and inhibitory interneurons of the neocortex (Galarreta and Hestrin, 1999, 2002; Gibson et al., 1999) remain coupled after the early postnatal period. This strik-
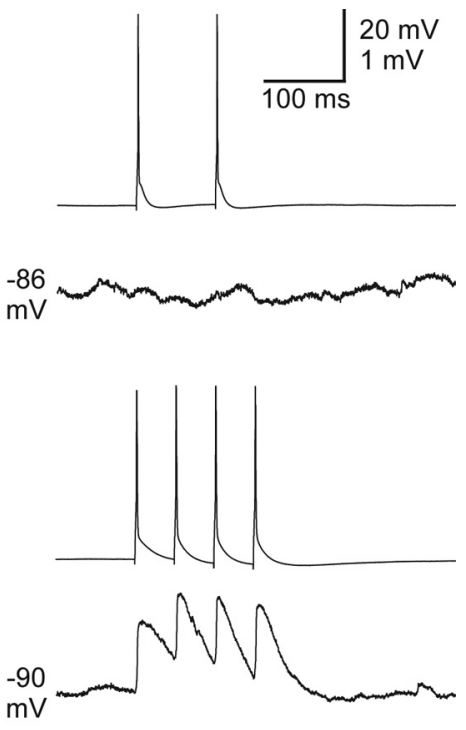

Figure 9. Chemical inhibitory synapses between TRN cells. $\boldsymbol{A}$, In a pair of P7 TRN cells, presynaptic action potentials (top)

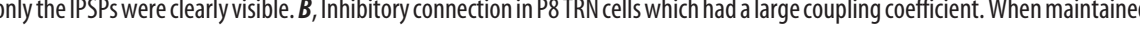
versed around $-90 \mathrm{mV}$ (bottom right). Inhibitory synapses between TRN cell pairs were rarely seen ( $2.8 \%$ incidence, $n=180$ possible connections) but existed as early as P4.

ing divergence suggests different functions for the gap junctions of immature and mature neurons in the thalamus.

Gap junction channels have been implicated in a variety of tasks, including intercellular signaling with electrical (ionic) current, passage of intercellular chemical messengers, signaling via chemicals released to the extracellular space through connexin hemichannels, and gap junction-mediated cell-cell adhesion (Kandler and Katz, 1995; Roerig and Feller, 2000; Elias and Kriegstein, 2008). Some or all of these functions may be important during the early development of the thalamus. However, the fact that functional measures of electrical coupling remain stable across development while gap junctional conductances dramatically increase suggests that ionic/electrical coupling rather than chemical/metabolic coupling may be more critical for TRN development. It has been hypothesized that gap junction coupling could influence chemical synaptogenesis by creating neuronal clusters that are synchronized; indeed, early gap junction coupling coincides with thalamocortical and corticothalamic synaptogenesis (Kandler and Katz, 1995). Once chemical synapses between thalamus and neocortex have been established, gap junctional coupling between pyramidal and VB cells begins to disappear (Connors et al., 1983; Lee et al., 2006). Unlike thalamic relay cells, TRN neurons remain well coupled after the first postnatal week by sharply increasing the efficacy of their gap junctions.

It seems plausible that gap junctions between TRN cells could be involved in organizing the topography of intra-TRN chemical synapses within the nucleus (Crabtree, 1996). Additionally, because gap junctions and inhibitory synapses between TRN cells may both be dendrodendritic (Liu and Jones, 2003; Pinault et al., 1997), there could be interactions between these two forms of communication during development. Gap junctions could assist in the formation or refinement of GABAergic synapses via metabolic and electrical coupling, especially at younger ages when 
depolarizing GABA-induced calcium transients could propagate through gap junctions into neighboring cells and exert second messenger effects. The tendency of electrically coupled neurons to synchronize their spiking will have consequences for spike timing, and perhaps spike timing-dependent synaptic plasticity. Conversely, depolarizing GABAergic transmission before P8 (Pangratz-Fuehrer et al., 2007) might be a signal by which gap junctions are upregulated between TRN cells.

Our results show that electrical synapses between TRN cells are capable of transmitting subthreshold signals fairly well, although high frequency transmission for connections between younger cells is considerably worse than that for more mature neurons. The age dependence of frequency transfer bandwidth could have consequences for rhythmic network activity in the thalamus. In the mature animal, spindle oscillations $(7-15 \mathrm{~Hz})$ are thought to be generated by reciprocal excitatory-inhibitory synaptic connections between neurons of the VB complex and the TRN (Huguenard and McCormick, 2007). The potential role of thalamic gap junctions in generating spindles is unclear (Fuentealba and Steriade, 2005), but it seems likely that electrical synapses in TRN play a role in synchronizing spindle rhythms within local spatial domains (Long et al., 2004). Electrical synapses in the mature TRN may also play a role in synchronizing more rapid thalamocortical oscillations, including those in the gamma range; mature $\mathrm{Cx} 36$ knock-out mice, which lack significant electrical coupling between neurons in both neocortex and TRN, display a deficiency in cortical gamma activity (Buhl et al., 2003). It had been suggested that thalamic theta-oscillations in vitro and cortical spindle waves in vivo do not occur in rodents before about P10 (Jouvet-Mounier et al., 1970; Warren et al., 1994). More recent work suggests, however, that spindle-like rhythms are common in cerebral cortex of neonatal rodents (Khazipov et al., 2004; Khazipov and Luhmann, 2006). The role of the TRN in the generation of these immature cortical rhythms has not been explored, but there is evidence that neonatal rhythms depend on neuronal gap junctions in neocortex (Dupont et al., 2006; Hanganu et al., 2009).

Neurons of the TRN are strongly coupled by gap junctions from birth to maturity. The stability of electrical coupling in immature TRN across development suggests that its gap junctions are closely regulated by homeostatic mechanisms, and that they play critical roles at all postnatal ages.

\section{References}

Agmon A, Connors BW (1991) Thalamocortical responses of mouse somatosensory (barrel) cortex in vitro. Neuroscience 41:365-379.

Altman J, Bayer SA (1988) Development of the rat thalamus: III. Time and site of origin and settling pattern of neurons of the reticular nucleus. J Comp Neurol 275:406-428.

Amitai Y, Gibson JR, Beierlein M, Patrick SL, Ho AM, Connors BW, Golomb D (2002) The spatial dimensions of electrically coupled networks of interneurons in the neocortex. J Neurosci 22:4142-4152.

Arumugam H, Liu X, Colombo PJ, Corriveau RA, Belousov AB (2005) NMDA receptors regulate developmental gap junction uncoupling via CREB signaling. Nat Neurosci 8:1720-1726.

Bennett MV (1966) Physiology of electrotonic junctions. Ann N Y Acad Sci 137:509-539.

Blethyn KL, Hughes SW, Crunelli V (2008) Evidence for electrical synapses between neurons of the nucleus reticularis thalami in the adult brain in vitro. Thalamus Relat Syst 4:13-20.

Bruzzone R, Dermietzel R (2006) Structure and function of gap junctions in the developing brain. Cell Tissue Res 326:239-248.

Buhl DL, Harris KD, Hormuzdi SG, Monyer H, Buzsaki G (2003) Selective impairment of hippocampal gamma oscillations in connexin-36 knockout mouse in vivo. J Neurosci 23:1013-1018.

Cachope R, Mackie K, Triller A, O’Brien J, Pereda AE (2007) Potentiation of electrical and chemical synaptic transmission mediated by endocannabinoids. Neuron 56:1034-1047.

Condorelli DF, Parenti R, Spinella F, Salinaro AT, Belluardo N, Cardile V, Cicirata F (1998) Cloning of a new gap junction gene (Cx36) highly expressed in mammalian brain neurons. Eur J Neurosci 10:1202-1208.

Connors BW, Long MA (2004) Electrical synapses in the mammalian brain. Annu Rev Neurosci 27:393-418.

Connors BW, Benardo LS, Prince DA (1983) Coupling between neurons of the developing rat neocortex. J Neurosci 3:773-782.

Crabtree JW (1996) Organization in the somatosensory sector of the cat's thalamic reticular nucleus. J Comp Neurol 366:207-222.

De Biasi S, Amadeo A, Arcelli P, Frassoni C, Spreafico R (1997) Postnatal development of GABA-immunoreactive terminals in the reticular and ventrobasal nuclei of the rat thalamus: a light and electron microscopic study. Neuroscience 76:503-515.

Deleuze C, Huguenard JR (2006) Distinct electrical and chemical connectivity maps in the thalamic reticular nucleus: potential roles in synchronization and sensation. J Neurosci 26:8633-8645.

Deschênes M, Madariaga-Domich A, Steriade M (1985) Dendrodendritic synapses in the cat reticularis thalami nucleus: a structural basis for thalamic spindle synchronization. Brain Res 334:165-168.

Dupont E, Hanganu IL, Kilb W, Hirsch S, Luhmann HJ (2006) Rapid developmental switch in the mechanisms driving early cortical columnar networks. Nature 439:79-83.

Elias LA, Kriegstein AR (2008) Gap junctions: multifaceted regulators of embryonic cortical development. Trends Neurosci 31:243-250.

Elias LA, Wang DD, Kriegstein AR (2007) Gap junction adhesion is necessary for radial migration in the neocortex. Nature 448:901-907.

Fuentealba P, Steriade M (2005) The reticular nucleus revisited: intrinsic and network properties of a thalamic pacemaker. Prog Neurobiol 75:125-141.

Fuentealba P, Crochet S, Timofeev I, Bazhenov M, Sejnowski TJ, Steriade M (2004) Experimental evidence and modeling studies support a synchronizing role for electrical coupling in the cat thalamic reticular neurons in vivo. Eur J Neurosci 20:111-119.

Galarreta M, Hestrin S (1999) A network of fast-spiking cells in the neocortex connected by electrical synapses. Nature 402:72-75.

Galarreta M, Hestrin S (2002) Electrical and chemical synapses among parvalbumin fast-spiking GABAergic interneurons in adult mouse neocortex. Proc Natl Acad Sci U S A 99:12438-12443.

Gibson JR, Beierlein M, Connors BW (1999) Two networks of electrically coupled inhibitory neurons in neocortex. Nature 402:75-79.

Gibson JR, Beierlein M, Connors BW (2005) Functional properties of electrical synapses between inhibitory interneurons of neocortical layer 4 . J Neurophysiol 93:467-480.

Hanganu IL, Okabe A, Lessmann V, Luhmann HJ (2009) Cellular mechanisms of subplate-driven and cholinergic input-dependent network activity in the neonatal rat somatosensory cortex. Cereb Cortex 19:89-105.

Huguenard JR, McCormick DA (2007) Thalamic synchrony and dynamic regulation of global forebrain oscillations. Trends Neurosci 30:350-356.

Huguenard JR, Prince DA (1992) A novel T-type current underlies prolonged $\mathrm{Ca}(2+)$-dependent burst firing in GABAergic neurons of rat thalamic reticular nucleus. J Neurosci 12:3804-3817.

Jahnsen H, Llinas R (1984) Electrophysiological properties of guinea-pig thalamic neurones: an in vitro study. J Physiol 349:205-226.

Jouvet-Mounier D, Astic L, Lacote D (1970) Ontogenesis of the states of sleep in rat, cat, and guinea pig during the first postnatal month. Dev Psychobiol 2:216-239.

Kandler K, Katz LC (1995) Neuronal coupling and uncoupling in the developing nervous system. Curr Opin Neurobiol 5:98-105.

Khazipov R, Luhmann HJ (2006) Early patterns of electrical activity in the developing cerebral cortex of humans and rodents. Trends Neurosci 29:414-418.

Khazipov R, Sirota A, Leinekugel X, Holmes GL, Ben-Ari Y, Buzsaki G (2004) Early motor activity drives spindle bursts in the developing somatosensory cortex. Nature 432:758-761.

Lam YW, Nelson CS, Sherman SM (2006) Mapping of the functional interconnections between thalamic reticular neurons using photostimulation. J Neurophysiol 96:2593-2600.

Landisman CE, Connors BW (2005) Long-term modulation of electrical synapses in the mammalian thalamus. Science 310:1809-1813.

Landisman CE, Long MA, Beierlein M, Deans MR, Paul DL, Connors BW 
(2002) Electrical synapses in the thalamic reticular nucleus. J Neurosci 22:1002-1009.

Lee SC, Cruikshank SJ, Connors BW (2006) Role of connexin36 in the electrical synapses between relay neurons in young developing thalamus. Soc Neurosci Abstr 32:132.12

Lee SH, Govindaiah G, Cox CL (2007) Heterogeneity of firing properties among rat thalamic reticular nuleus neurons. J Physiol 582:195-208.

Liu XB, Jones EG (2003) Fine structural localization of connexin-36 immunoreactivity in mouse cerebral cortex and thalamus. J Comp Neurol 466:457-467.

Long MA, Landisman CE, Connors BW (2004) Small clusters of electrically coupled neurons generate synchronous rhythms in the thalamic reticular nucleus. J Neurosci 24:341-349.

Lo Turco JJ, Kriegstein AR (1991) Clusters of coupled neuroblasts in embryonic neocortex. Science 252:563-566.

Mitrofanis J, Baker GE (1993) Development of the thalamic reticular and perireticular nuclei in rats and their relationship to the course of growing corticofugal and corticopetal axons. J Comp Neurol 338:575-587.

Montoro RJ, Yuste R (2004) Gap junctions in developing neocortex: a review. Brain Res Brain Res Rev 47:216-226.

Moody WJ, Bosma MM (2005) Ion channel development, spontaneous activity, and activity-dependent development in nerve and muscle cells. Physiol Rev 85:883-941.

Moreno AP, Lau AF (2007) Gap junction channel gating modulated through protein phosphorylation. Prog Biophys Mol Bio 94:107-119.

Ohara PT (1988) Synaptic organization of the thalamic reticular nucleus. J Electron Microsc Tech 10:283-292.
Ortino B, Inverardi F, Morante-Oria J, Fairen A, Frassoni C (2003) Substrates and routes of migration of early generated neurons in the developing rat thalamus. Eur J Neurosci 18:323-332.

Pangratz-Fuehrer S, Rudolph U, Huguenard JR (2007) Giant spontaneous depolarizing potentials in the developing thalamic reticular nucleus. J Neurophysiol 97:2364-2372.

Pinault D, Smith Y, Deschênes M (1997) Dendrodendritic and axoaxonic synapses in the thalamic reticular nucleus of the adult rat. J Neurosci 17:3215-3233.

Roerig B, Feller MB (2000) Neurotransmitters and gap junctions in developing neural circuits. Brain Res Brain Res Rev 32:86-114.

Rörig B, Sutor B (1996) Regulation of gap junction coupling in the developing neocortex. Mol Neurobiol 12:225-249.

Sanchez-Vives MV, Bal T, McCormick DA (1997) Inhibitory interactions between perigeniculate GABAergic neurons. J Neurosci 17:8894-8908.

Spitzer NC, Ribera AB (1998) Development of electrical excitability in embryonic neurons: mechanisms and roles. J Neurobiol 37:190-197.

Warren RA, Jones EG (1997) Maturation of neuronal form and function in a mouse thalamo-cortical circuit. J Neurosci 17:277-295.

Warren RA, Agmon A, Jones EG (1994) Oscillatory synaptic interactions between ventroposterior and reticular neurons in mouse thalamus in vitro. J Neurophysiol 72:1993-2003.

Yuste R, Peinado A, Katz LC (1992) Neuronal domains in developing neocortex. Science 257:665-669.

Zsiros V, Maccaferri G (2008) Noradrenergic modulation of electrical coupling in GABAergic networks of the hippocampus. J Neurosci 28:18041815 . 